\title{
Information Management Workflow and Tools Enabling Multiscale Modeling Within ICME Paradigm
}

\author{
Steven M. Arnold*, Brett A. Bednarcyk ${ }^{\dagger}$, \\ NASA Glenn Research Center, Cleveland, $\mathrm{OH} 44135$ \\ Nic Austin*, Igor Terentjev ${ }^{\S}$, Dave Cebon ${ }^{* *}$ and Will Marsden ${ }^{\dagger \dagger}$ \\ Granta Design, Ltd., Cambridge, UK
}

\begin{abstract}
With the increased emphasis on reducing the cost and time to market of new materials, the need for analytical tools that enable the virtual design and optimization of materials throughout their processing - internal structure - property - performance envelope, along with the capturing and storing of the associated material and model information across its lifecycle, has become critical. This need is also fueled by the demands for higher efficiency in material testing; consistency, quality and traceability of data; product design; engineering analysis; as well as control of access to proprietary or sensitive information. Fortunately, material information management systems and physics-based multiscale modeling methods have kept pace with the growing user demands. Herein, recent efforts to establish workflow for and demonstrate a unique set of web application tools for linking NASA GRC's Integrated Computational Materials Engineering (ICME) Granta MI ${ }^{\circledR}$ database schema and NASA GRC's Integrated multiscale Micromechanics Analysis Code (ImMAC) software toolset are presented. The goal is to enable seamless coupling between both test data and simulation data, which is captured and tracked automatically within Granta MI ${ }^{\circledR}$, with full model pedigree information. These tools, and this type of linkage, are foundational to realizing the full potential of ICME, in which materials processing, microstructure, properties, and performance are coupled to enable application-driven design and optimization of materials and structures.
\end{abstract}

\section{Introduction}

$\mathrm{W}$ ith the increased emphasis on reducing the cost and time to market of new materials, ICME (Integrated Computational Materials Engineering) has become a fast growing discipline within materials science and engineering. The vision of ICME is compelling in many respects, not only for the value added in reducing time to market for new products with advanced, tailored materials, but also for enhanced efficiency and performance of these materials. Although the challenges and barriers (both technical and cultural) are formidable, substantial cost, schedule, and technical benefits can result from broad development, implementation, and validation of ICME principles ${ }^{[1]}$. ICME is an integrated approach to the design of products, and the materials that comprise them, by linking material and structural models at multiple time and length scales.

A key ingredient is the linkage with manufacturing processes, which produce internal material structures, and in turn influence material properties and allowables, enabling tailoring (engineering) of materials to specific industrial applications. Figure 1 illustrates the interconnection of these scales and their cause/effect relationships, e.g., processing conditions produce a particular microstructure from which properties are obtained, which then influence the specific structural performance. Note that the evolution of elliptical line types (i.e., dotted to dashed to solid line) is purposely included to imply the level of maturity/understanding (from immature, to semi-mature, to mature, respectively) of modeling at each level of scale (both temporal and geometric). Furthermore, the figure illustrates the difference between two non-exclusive viewpoints; that is designing "with-the-material" (structural analyst viewpoint) versus designing "the material" (materials scientist viewpoint). It is also apparent that the fundamental linkage between these two viewpoints is ultimately the associated constitutive model(s) for a particular material. It is critical

\footnotetext{
*Technical Lead: Multiscale Modeling, Structures and Materials Division, 21000 Brookpark Road, AIAA member.

${ }^{\dagger}$ Multiscale Modeling and Multiphysics Branch, 21000 Brookpark Road, AIAA Associate Fellow.

${ }^{\dagger}$ Lead Software Architect, Software Division, Rustat House, 62 Clifton Road, Cambridge, UK. CB17EG.

${ }^{\S}$ Senior Engineer, Lead Software, Software Division, Rustat House, 62 Clifton Road, Cambridge, UK. CB17EG.

${ }^{* *}$ Technical Director, Rustat House, 62 Clifton Road, Cambridge, UK. CB17EG.

${ }^{\dagger}$ Director of Industry Relations, Rustat House, 62 Clifton Road, Cambridge, UK. CB17EG.
} 
to understand the input and output at each scale in order to determine the appropriate "handshaking" between scales and the meaningful properties that are ultimately required by a structural analyst.

Equally important is the fact that experiments (whether computational/virtual or laboratory) performed at a given level can be viewed from two perspectives. If one "looks up" to higher scales, then the results can be viewed as exploration or characterization experiments used to identify/obtain the necessary model features or parameters, respectively, operating at the present and/or next higher level. Conversely, if one "looks down", these same results can be used to validate the modeling methods/approaches employed to transition from the lower level(s) to the given level.

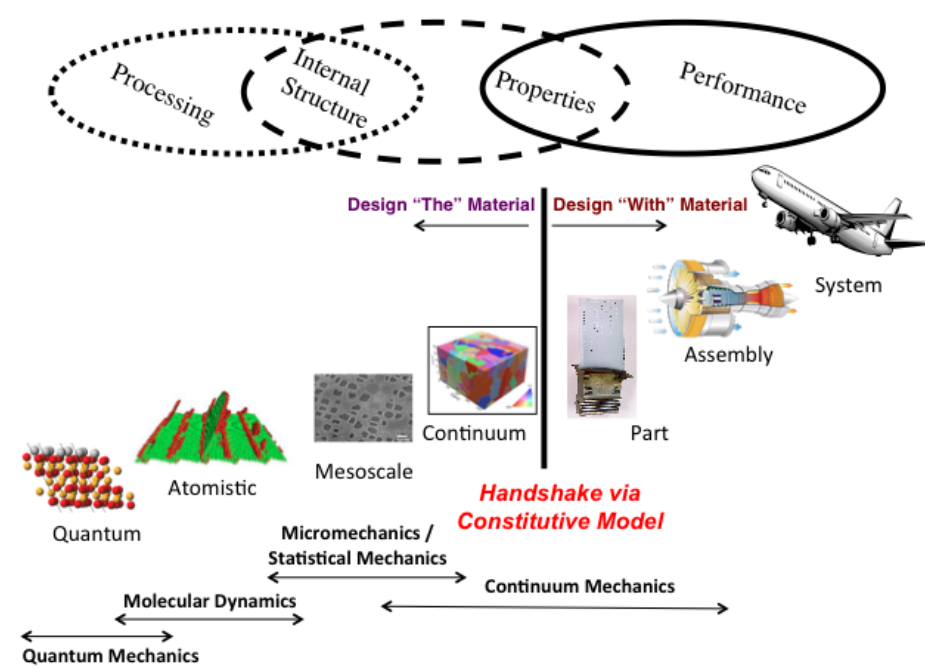

Figure 1 Description of associated length scale dependence and modeling methods in the context of ICME.

While there is a clear indication that ICME is growing, utilization of ICME in the daily work of researchers and engineers is still lacking. Key contributing factors, given that ICME is an inherently data intensive activity, are the lack of a robust information management system and the lack of a digital storage culture within most organizations. This stems from the fact that, on the surface, a materials properties database may seem simply like an elaborate means of storing, retrieving and distributing materials data; something akin to an electronic file cabinet. However, as discussed by Marsden et al. ${ }^{[2]}$ and Arnold et al. ${ }^{[3]}$, an effective ICME materials database (e.g., one in which experimental and computational mechanics are fully coupled) must allow the data inside the database to be easily accessible by analysis tools and allow the results from analyses to be read back into the database and stored with all of the associated metadata, while keeping track of associations across the full range of length scales.

For example, a physics-based model to predict the yield strength of a nickel-based superalloy may need to draw upon quantum mechanics predictions of stacking fault energies, lattice distortions, and phase equilibria of several different alloying elements. These predictions might be combined with microstructural scale models that either use the quantum mechanics predictions or are calibrated with experimental data. Phase equilibria (e.g., CALPHAD ${ }^{\circledR}$ ) models are an example, as are processing-microstructure models of castings or forgings. Important information necessary for a yield strength prediction would include not only equilibrium phases but also the kinetics of microstructural evolution (of several features, including $\gamma^{\prime}$ precipitate and carbide size and spacing, grain size and grain boundary phases). The maturity of these models already allows semi-quantitative predictions of various parameters, but development of higher fidelity models will require the capture, analysis and dissemination of higher fidelity data, as well as all associated pedigree information for calibration and validation. For example, while a current model may utilize an average particle size as a key parameter, future models may require entire particle size and shape distributions to be measured and tracked with respect to various manufacturing methods. Clearly, the enormity of data types (e.g., discrete, functional, structured, and unstructured) and the sheer quantity of data can be overwhelming. Consequently, historical static data systems are likely to be gradually phased out, evolving to become an integral part of dynamic materials property databases that are web-accessible and in which data - and the relationships between data - can be interactively searched, reorganized, analyzed, and applied. These dynamic databases have great superiorities in satisfying the needs of modern materials-related sciences and engineering focused activities like ICME. 
It is also critical to understand that ICME is not just developing processing-microstructure (P-M) relationships or microstructure-property (M-P) relationships independently, rather it is the full integration of these various length scale-specific relationships, wherein linkages from processing all the way up to performance can be made and utilized. This requirement greatly increases the need for data/metadata and contextual linkage so that knowledge can be both captured and discovered. For example, the variety and complexity of modern materials and their applications necessitate complicated, and often extensive, materials testing. As for composite materials, large volumes of test data on various forms of the composites themselves, as well as individual constituents' thermal and mechanical behavior, are often required. Given a micromechanics based analysis approach, it is typical to require that data for each constituent be reliably and conveniently traced back from the final products through their processing steps to the original raw materials. A second example is the need to provide adequate data to support increasingly sophisticated nonlinear, anisotropic and multi-scale engineering analyses. Here again, instead of storing a simple set of reduced, point-wise data, like elastic modulus and yield strength, the entire response (e.g., stress-strain, creep, relaxation, etc.) curves may be required. Collating, storing, processing, interacting with, and finally applying such data and metadata requires advanced dynamic information systems, enabling management of changing proprietary data alongside reference data collections, while ensuring consistency, quality, applicability and traceability.

Prior publications ${ }^{[3-6]}$ discussed the data scheme, best practices, and informatics required to establish a robust, $21^{\text {st }}$ century, information management system for capturing and analyzing material information. The goal of the information management system is to enable: 1) generalized constitutive modeling and 2) data-mining to establish microstructure/property/failure relationships for monolithic and composite materials. The proposed schema/ requirements for ICME were first demonstrated using a turbine disk Ni-based superalloy, by Arnold et al. ${ }^{[3]}$. Then, Arnold et al. ${ }^{[5,6]}$ argued that integrating both virtual (computationally based) and experimental data, over the entire material data life cycle (see Figure 2) and at various length scales, in the same information management system was essential for ICME to become a reality and to permeate the materials and engineering cultures within a given organization. The proposed ICME schema, which has been adopted by the MDMC ${ }^{\sharp 1}$, is given in Figure 3. The specifics of the schema (i.e., required attributes) and the format (e.g., attribute type and record layout) for best storing such information were discussed in detail in Arnold et al. ${ }^{[5,6]}$ for storing monolithic and composite material information at the coupon level. In the case of monolithic materials (e.g., fiber and matrix), three tables and their associated attributes were defined to enable the complete data life cycle to be handled, these are the: Deformation Table, Damage-Life Table, and Software Tools Table, see Figure 3. Whereas, in the case of composite materials one must think more broadly as multiple length scales can be involved depending upon the approach taken (i.e., macromechanics or micromechanics) to define the material's "constitutive model". Consequently, the additional meso or macro scale above the constituent scale (e.g., that associated with monolithic material) necessitated the introduction of a fourth table, the Composite Table. Clearly, extension to other scales (e.g., atomistic, processing, microstructure modeling, structural) may require either the addition of new tables with appropriate attributes to the Model pedigree group within Figure 3 (e.g., Process Model Table) or new scale specific attributes to represent each new scale considered. As an example, Figure 4 illustrates the interaction between experimental data and virtual data (data resulting from simulation tools) in that some experimental processing data (A) serves as input to a process model which in turn outputs some microstructural feature (W), which is stored in the database. This virtual microstructure data is then combined with measured microstructural data (B) and provided as input to a micromechanics and/or statistical mechanics analysis package, which then generates material property data (X, Y), which again is stored in the database. This property data $(\mathrm{X}, \mathrm{Y})$ is then subject to experimental validation (E,F), and also used in some continuum level analysis package (e.g., finite element analysis (FEA)) to assess some performance criteria (e.g., fatigue life, creep rupture, buckling load) Z; which is again stored in the database. Clearly, the present schema (with its assembly of model pedigree tables) not only allows model information and model parameters to be stored in a location that is easily accessible by FEA or other analysis codes through some type of interface software (e.g., Granta Material Gateway ${ }^{\circledR}$ ), but also stores any associated simulation data necessary to assist in the evaluation, verification and validation of model output and certification of toolsets at multiple length scales. Also, once all of the input/output protocols are established, it can enable the seamless integration of these toolsets with optimization (e.g., OpenMDAO ${ }^{[7]}$ ) algorithms that will provide the final linkage of processing to performance criteria-thus realizing true ICME.

\footnotetext{
\$The Material Data Management Consortium (MDMC) is a group of aerospace and energy sector organizations (both industrial and governmental) that have joined forces to develop best practices and associated software tools to integrate material and structural information technology with the realities of practical product design and advanced research. This group was established in 2002 through collaboration between ASM International, NASA Glenn Research Center and Granta Design Limited ${ }^{[8]}$, see www.mdmc.net ${ }^{[9]}$.
} 


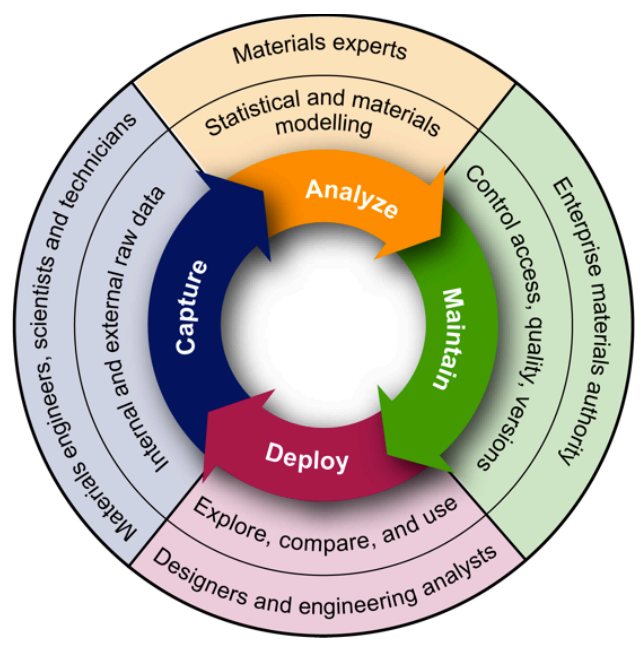

Figure 2 Four aspects of material data lifecycle as defined by the MDMC.

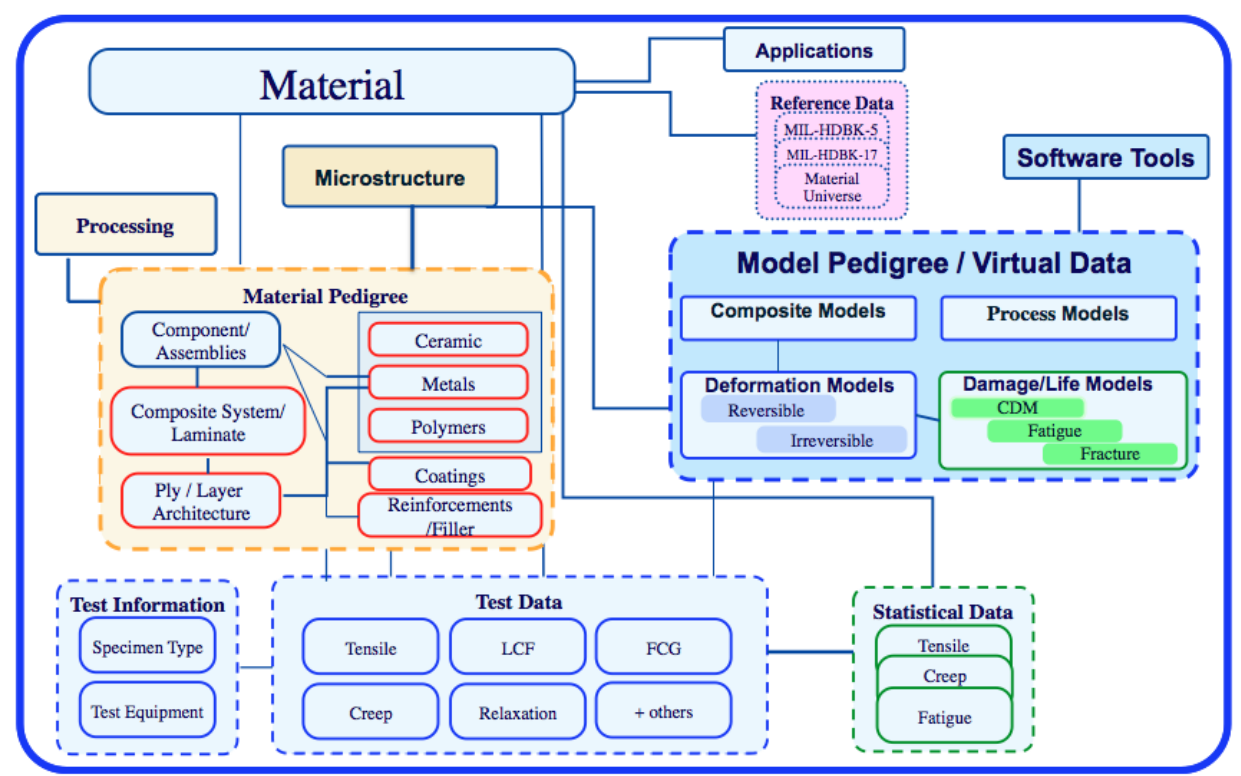

Figure 3 NASA Glenn's Schema Modified to Incorporate Virtual data to enable ICME 


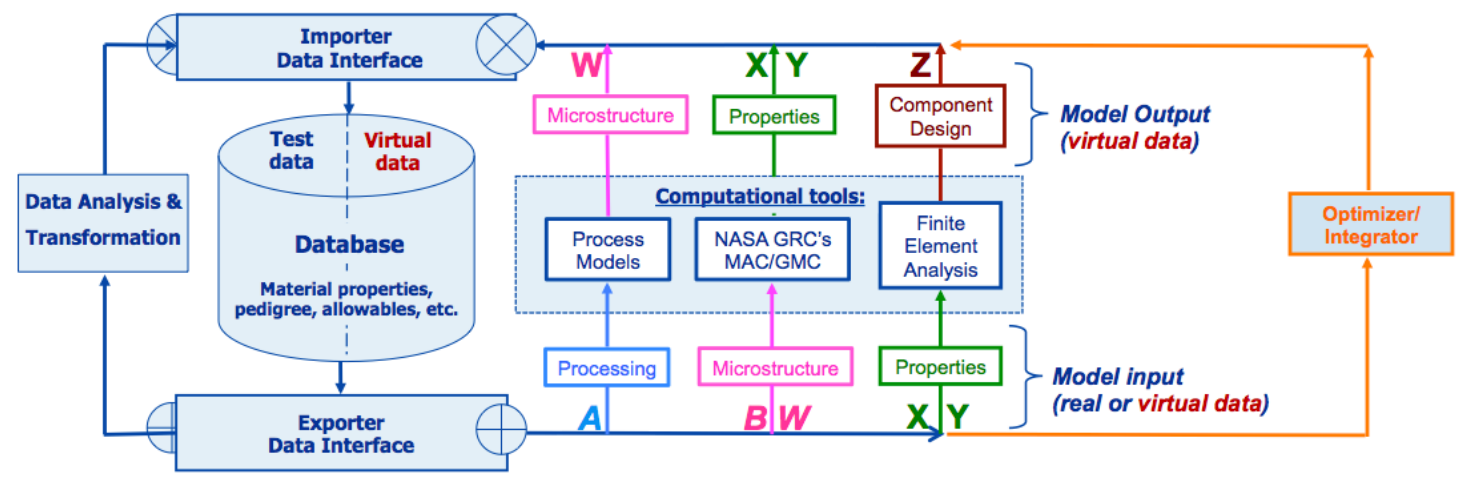

Figure 4 ICME infrastructure for housing modeling and testing information. Private communications with Dr. David Cebon, Cambridge University and Granta Design Ltd., 2013.

In the remainder of this paper, the next step toward the envisioned robust ICME information management system is described. The workflow and associated web applications, which enable linkage of third party model simulation software (with full model pedigree storage) to the information management system, are described. NASA GRC's MAC/GMC micromechanics analysis software serves as the example third party modeling software, with the linkage to the Granta MI information management system described. This linkage exercises and validates the schema for the first time on two levels of scale. On the micro scale, composite constituent materials from the Granta MI database are provided as input to MAC/GMC. The web applications execute the requested MAC/GMC simulations, and the results (representing the composite at the macro scale), along with all metadata and model pedigree information, are then captured and stored in the database. The micromechanics model, along with the newly developed workflow and web applications, are described below.

\section{Micromechanics of Composite Materials and Structures}

In its broadest context, a composite is anything comprised of two or more entities with a recognizable interface (i.e., distinct internal boundaries) between them. If these internal boundaries are ignored, continuum mechanics can be used to model composite materials as pseudo-homogenous, anisotropic materials, with directionally dependent "effective," "homogenized," or "smeared" material properties. Micromechanics, on the other hand, attempts to account for the internal boundaries within a composite material and capture the effects of the composite's internal arrangement. In micromechanics, the individual materials (typically referred to as constituents or phases) that make up a composite are each treated as continua via continuum mechanics models, with their individual properties and arrangement dictating the overall behavior of the composite material. Over the past two decades NASA Glenn Research Center has been developing the ImMAC suite of tools for analyzing continuous, discontinuous, woven, and smart (piezo-electo-magnetic) composite materials and/or structures composed of such materials. MAC/GMC (a comprehensive and versatile stand-alone micromechanics analysis computer code), HyperMAC (the coupling of MAC/GMC micromechanics with the commercial structural sizing software known as HyperSizer ${ }^{[10]}$ ), MSGMC (the recursive coupling of micromechanics with micromechanics, for woven composites), and FEAMAC (the coupling of MAC/GMC micromechanics with the commercial finite element code, Abaqus ${ }^{[1]}$ ) make up this suite. At the core of these various tools is the well-known method of cells family of micromechanics theories (e.g., Method of Cells, Generalized Method of Cells, and High-Fidelity Generalized Method of Cells) developed by Aboudi and co-workers ${ }^{[12]}$. These methods provide semi-closed form solutions for determining global anisotropic composite properties in terms of the constituent material properties and arrangement, while also providing the full three dimensional stresses and strains in each of the constituent subcells. For a detailed, comprehensive discussion on modeling of composite materials, the reader is referred to the book entitled Micromechanics of Composite Materials: A Generalized Multiscale Analysis Approach ${ }^{[12]}$. Micromechanics based analysis lends itself to ICME in that it can link the processing and microstructure of the composite material directly to the resulting properties and performance of the material/structure, thereby enabling the engineer to not only "design-with-the" material but also, concurrently, 
"design-the" material. Consequently, by developing a database schema capable of handling a micromechanics approach enables demonstration of an ICME capable (multiscale) database framework for composite materials.

\section{A. The Generalized Method of Cells (GMC)}

The Generalized Method of Cells (GMC) micromechanics theory assumes that a continuously reinforced composite microstructure can be represented as a collection of periodic repeating unit cells (RUCs) containing an arbitrary number of constituents, as illustrated in Figure 5. The RUC (indicated by a dashed line in Figure 5) is then discretized into $N_{\beta}$ x $N_{\gamma}$ rectangular subcells (in the case of doubly periodic GMC), as exhibited in Figure 6. Note, triply-periodic microstructures (e.g., particulate reinforced or 3D woven composites) although not discussed here, can also be represented by GMC. Each of these subcells is occupied by one of the constituent materials (e.g., SiC Fiber, $\mathrm{BN}$ coating, $\mathrm{SiC}$ matrix, and Free $\mathrm{Si}$ in the case of $\mathrm{SiC} / \mathrm{SiC}$ composites). The number of subcells and the number of materials are completely general. In GMC, a first-order displacement field within the subcells is assumed, and displacement and traction continuity conditions are enforced in an average, integral sense at the subcell interfaces of the discretized RUC. These continuity conditions are used to formulate a set of semi-analytical linear algebraic equations that are solved for the local strains in each subcell $(\beta \gamma)$ in terms of globally applied strains or stresses. Then, local constitutive laws can be utilized to obtain the local stresses in subcell $(\beta \gamma)$,

$$
\begin{gathered}
\boldsymbol{\varepsilon}^{(\beta \gamma)}=\mathbf{A}^{(\beta \gamma)} \overline{\boldsymbol{\varepsilon}}+\mathbf{D}^{(\beta \gamma)}\left(\boldsymbol{\varepsilon}_{s}^{I}+\boldsymbol{\varepsilon}_{s}^{T}\right) \\
\boldsymbol{\sigma}^{(\beta \gamma)}=\mathbf{C}^{(\beta \gamma)}\left\{\mathbf{A}^{(\beta \gamma)} \overline{\boldsymbol{\varepsilon}}+\mathbf{D}^{(\beta \gamma)}\left(\boldsymbol{\varepsilon}_{s}^{I}+\boldsymbol{\varepsilon}_{s}^{T}\right)-\left(\boldsymbol{\varepsilon}^{I(\beta \gamma)}+\boldsymbol{\varepsilon}^{T(\beta \gamma)}\right)\right\}
\end{gathered}
$$

where $\boldsymbol{\sigma}$ is the stress tensor, $\mathbf{C}$ is the stiffness tensor, and $\boldsymbol{\varepsilon}, \boldsymbol{\varepsilon}^{I}$, and $\boldsymbol{\varepsilon}^{T}$ are the total, inelastic, and thermal strain tensors, respectively, $\boldsymbol{\varepsilon}_{s}^{I}$ and $\boldsymbol{\varepsilon}_{s}^{T}$ are 6 by $\mathrm{N}_{s} \mathrm{~N}_{r}$ matrices containing all components of the inelastic and thermal strains, respectively, of every subcell (appropriately ordered), $\mathbf{A}^{\left(\beta_{r}\right)}$ is the strain concentration tensor, and $\mathbf{D}^{\left(\beta_{\mu}\right)}$ is the thermo-inelastic strain concentration tensor. $(\beta \gamma)$ superscripts denote subcell quantities, whereas overbars indicate global (effective, homogenized composite) quantities. The generalized constitutive law for the effective, homogenized composite can be formulated as,

$$
\overline{\boldsymbol{\sigma}}=\mathbf{C}^{*}\left(\overline{\boldsymbol{\varepsilon}}-\overline{\boldsymbol{\varepsilon}}^{I}-\overline{\boldsymbol{\varepsilon}}^{T}\right)
$$

where the effective stiffness tensor, $\mathbf{C}^{*}$, effective inelastic strains, $\overline{\boldsymbol{\varepsilon}}^{I}$, and effective thermal strains, $\overline{\boldsymbol{\varepsilon}}^{T}$, are given by,

$$
\begin{gathered}
\mathbf{C}^{*}=\frac{1}{h l} \sum_{\beta=1}^{N_{\beta}} \sum_{\gamma=1}^{N_{\gamma}} h_{\beta} l_{\gamma} \mathbf{C}^{(\beta \gamma)} \mathbf{A}^{(\beta \gamma)} \\
\overline{\boldsymbol{\varepsilon}}^{I}=-\frac{\mathbf{C}^{*-1}}{h l} \sum_{\beta=1}^{N_{\beta}} \sum_{\gamma=1}^{N_{\gamma}} h_{\beta} l_{\gamma} \mathbf{C}^{(\beta \gamma)}\left(\mathbf{D}^{(\beta \gamma)} \boldsymbol{\varepsilon}_{s}^{I}-\boldsymbol{\varepsilon}^{I(\beta \gamma)}\right) \\
\overline{\boldsymbol{\varepsilon}}^{T}=-\frac{\mathbf{C}^{*-1}}{h l} \sum_{\beta=1}^{N_{\beta}} \sum_{\gamma=1}^{N_{\gamma}} h_{\beta} l_{\gamma} \mathbf{C}^{(\beta \gamma)}\left(\mathbf{D}^{(\beta \gamma)} \boldsymbol{\varepsilon}_{s}^{T}-\boldsymbol{\varepsilon}^{T(\beta \gamma)}\right)
\end{gathered}
$$

$h_{\beta}$ and $l_{r}$ are the dimensions of the subcells, $h$ and $l$ are the dimensions of the RUC, and $\overline{\boldsymbol{\sigma}}$ and $\overline{\boldsymbol{\varepsilon}}$ are the effective (homogenized) stress and strain tensors. Extensive details regarding this derivation can be found in Aboudi et al. $(2013)^{[12]}$. 


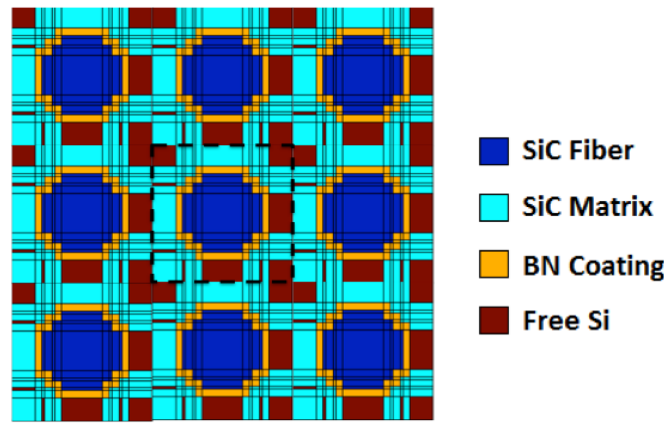

Figure 5 Representation of a doubly-periodic microstructure of a CMC composite material.

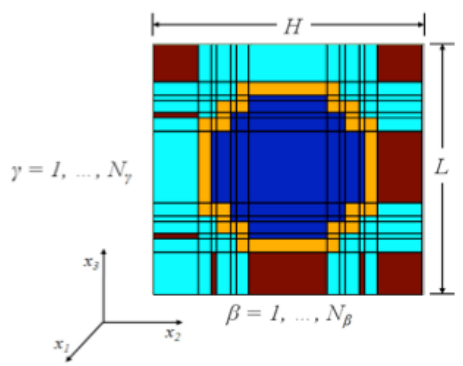

Figure 6 Discretization of a doubly-periodic RUC.

The developed ICME database schema is sufficiently general to admit all data associated with a micromechanics theory such as GMC. In the present schema the various constitutent constitutive model parameters, be they reversible or irreversible, are stored in the individual folders/records associated with specific materials and constitutive models, for each material, in the Deformation Table. Whereas, the effective composite properties, $\mathbf{C}^{*}$, and effective stress and strain responses, denoted by the overbar terms in equations (3), (5), and (6), and associated metadata (e.g., RUC details and simulation input file) are stored in the Composite Table. The specific attributes and associated data types, as well as the corresponding layout of information for the Deformation Table and Composite Table (see Table 1) are given in Arnold et al [5,6]. 
Table 1 Layout and Attributes for Composite Model Table

\begin{tabular}{|c|c|}
\hline Attributes & Type \\
\hline \multicolumn{2}{|l|}{ Project Information } \\
\hline Performing Organization & STXT \\
\hline Project Name/Funding Source & STXT \\
\hline Point of Contact (POC) & STXT \\
\hline \multicolumn{2}{|l|}{ Material Description } \\
\hline Material & STXT \\
\hline Material Class & DCT \\
\hline Commercial Name & STXT \\
\hline Specific Name & STXT \\
\hline Material Pedigree Record & Link \\
\hline Batch Number & STXT \\
\hline Material Notes & LTXT \\
\hline \multicolumn{2}{|l|}{ General Modeling Information } \\
\hline Model ID & STXT \\
\hline Characterization/Analysis Date & DAT \\
\hline Temperature & PNT \\
\hline Temperature Range & RNG \\
\hline Assumptions & LTXT \\
\hline \multicolumn{2}{|l|}{ Micromechanics Modeling Information } \\
\hline Micromechanics Method & DCT \\
\hline Micromechanics Analysis Tool & STXT \\
\hline Micromechanics Tool Information & Link \\
\hline Micromechanics Input File & FIL \\
\hline No. of Constiutents & INT \\
\hline RUC/RVE Constituitve Description & TABL \\
\hline RUC/RVE Image & PIC \\
\hline Fiber Packing Arrangement & DCT \\
\hline Effective Thermo-Elastic Composite Properties & TABL \\
\hline Micromechanics Notes & LTXT \\
\hline \multicolumn{2}{|l|}{ Laminate Level Modeling Information } \\
\hline Laminate Name & STXT \\
\hline Laminate Specification & STXT \\
\hline Architecture Type & DCT \\
\hline Laminate Pattern & DCT \\
\hline Laminate Thickness & PNT \\
\hline Ply Thickness (avg) & PNT \\
\hline No. of Plies & INT \\
\hline Laminate Definition & TABL \\
\hline Laminate Analysis Tool & STXT \\
\hline Laminate Analysis Tool Information & Link \\
\hline Composite Laminate Analysis Input File & FIL \\
\hline Laminate Notes & LTXT \\
\hline Laminate Extensional Stiffness Matrix (A) & TABL \\
\hline Laminate Coupling Stiffness Matrix (B) & TABL \\
\hline Laminate Bending Stiffness Matrix (D) & TABL \\
\hline \multicolumn{2}{|l|}{ Volume Fractions } \\
\hline Total Matrix Volume Fraction & PNT \\
\hline Total Reinforcement Volume Fraction & PNT \\
\hline Total Void/Porosity Voume Fraction & PNT \\
\hline
\end{tabular}

\begin{tabular}{|c|c|}
\hline Attributes & Type \\
\hline \multicolumn{2}{|l|}{ Multiscale Modeling Information } \\
\hline Multiscale Analysis Tool & DCT \\
\hline Multiscale Analysis Tool Information & Links \\
\hline Multiscale Analysis Input File & FIL \\
\hline Multiscale Modeling Notes & LTXT \\
\hline \multicolumn{2}{|l|}{ Composite Test Data Used for Characterization/Vaildation } \\
\hline Tensile Test Data (Linked Records location in layout) & Links \\
\hline Creep Test Data (Linked Records location in layout) & Links \\
\hline RelaxationTest Data (Linked Records location in layout) & Links \\
\hline Cyclic Test Data (Linked Records location in layout) & Links \\
\hline Generic Test Data (Linked Records location in layout) & Links \\
\hline \multicolumn{2}{|l|}{ Simulation Responses } \\
\hline Stress vs. Strain Response (11 axis) & FDA \\
\hline Stress vs. Strain Response (22 axis) & FDA \\
\hline Stress vs. Strain Response (33 axis) & FDA \\
\hline Stress (11 axis) vs. Time & FDA \\
\hline Stress ( 22 axis) vs. Time & FDA \\
\hline Stress ( 33 axis) vs. Time & FDA \\
\hline Total Strain (11 axis) vs. Time & FDA \\
\hline Total Strain ( 22 axis) vs. Time & FDA \\
\hline Total Strain (33 axis) vs. Time & FDA \\
\hline Shear Stress vs. Shear Strain Response (12 axis) & FDA \\
\hline Shear Stress vs. Shear Strain Response (13 axis) & FDA \\
\hline Shear Stress vs. Shear Strain Response (23 axis) & FDA \\
\hline Shear Stress (12 axis) vs. Time & FDA \\
\hline Shear Stress (13 axis) vs. Time & FDA \\
\hline Shear Stress ( 23 axis) vs. Time & FDA \\
\hline Total Shear Strain (12 axis) vs. Time & FDA \\
\hline Total Shear Strain (13 axis) vs. Time & FDA \\
\hline Total Shear Strain ( 23 axis) vs. Time & FDA \\
\hline Force Resultant vs. Midplane normal strain (xx-axis) & FDA \\
\hline Force Resultant vs. Midplane normal strain (yy-axis) & FDA \\
\hline Force Resultant vs. Midplane normal strain (xy-axis) & FDA \\
\hline Moment Resultant vs. Midplane curvature (xx-axis) & FDA \\
\hline Moment Resultant vs. Midplane curvature (yy-axis) & FDA \\
\hline Moment Resultant vs. Midplane curvature (xy-axis) & FDA \\
\hline \multicolumn{2}{|l|}{ References } \\
\hline General Modeling Notes & LTXT \\
\hline Model References & LTXT \\
\hline
\end{tabular}

DCT Discrete Text (specified choices)

FDA Functional Data Attribute (with associated parameters)

FIL Allows the association of any file type to a given record

INT Integer Value

LOG Logical

LTXT Long Text Field

PIC Allows association of any image format to a given record

PNT Point value

RNG Range variable

STXT Short Text Field

TABL Tabular Attribute (multiple columns of data - PNT, STXT, DCT, INT, link)

\section{B. FEAMAC Multiscale Framework}

A key ingredient of ICME is the linkage of the subscale (lower length scale) effects to structural performance. As such, a synergistic multiscale framework (which executes concurrent multiscaling in time, but sequential multiscaling in space ${ }^{[13]}$ ) has been constructed to simulate the nonlinear response of composite structures by modeling the fibermatrix architecture as an RUC at the microscale using GMC and coupling the microscale to a lamina/laminate level (macroscale) finite element model (FEM). The commercial finite element software, Abaqus ${ }^{[1]]}$, is used as the FEM platform, and the MAC/GMC core micromechanics software ${ }^{[14,15]}$ is used to perform microscale calculations. The scales are linked using the FEAMAC software implementation ${ }^{[16]}$, which utilizes various Abaqus/Standard user subroutines. A schematic displaying a typical multiscale model using FEAMAC is displayed in Figure 7. The strains at the FEM integration point are applied to the RUC and the local subcell fields are determined using GMC (this 
process is referred to as localizaion). If the subcell material behavior is nonlinear, the local stresses and strains are used to calculate the local stiffnesses, inelastic strains, thermal strains, and/or state variables via the local constituent constitutive laws. Homogenization of the RUC is then performed to obtain the global stiffnesses, inelastic strains, thermal strains, and/or state variables. The global stresses at the integration point are then calculated using these global, homogenized fields, and the effects of any nonlinear subscale phenomena are introduced into the macroscale through changes in the integration point stress state and stiffness. The global stresses, material Jacobian, and updated state variables at each FEM integration point are then supplied to Abaqus through the user material UMAT subroutine. For complete details on the FEAMAC implementation, the reader is referred to Bednarcyk and Arnold ${ }^{[15]}$ and Aboudi et al. ${ }^{[11]}$

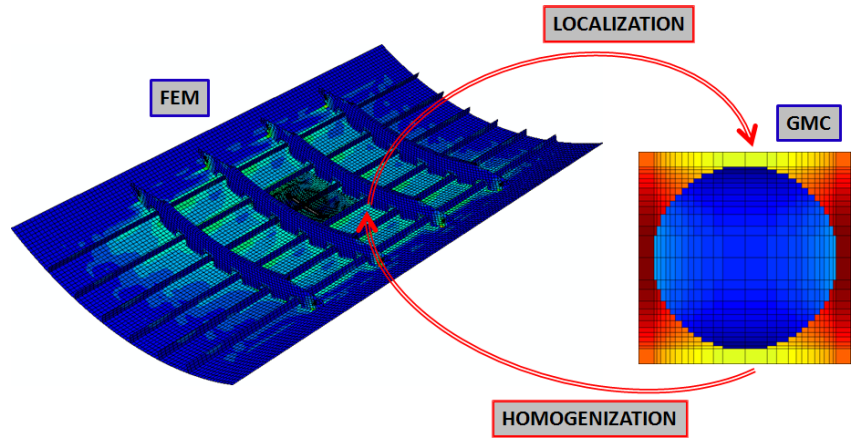

Figure 7 Diagram showing coupling of macroscale FEM and microscale GMC models.

With the ability to link the GMC micromechanics model, which accounts for processing and microstructure while predicting properties (as discussed in the previous section), with a structural FEM, which simulates performance, the full range of ICME-related scales (Figures 1 and 4) have been captured. Note the exact database location of the simulation results of the full structural analysis (i.e., stiffened panel in Figure 7) has yet to be finalized as they could reside in a model pedigree table, the application table, or in a product life management (PLM) system external to the Granta MI database. More specific details regarding layout and associated attributes within the composite and software tables are given in the next section.

\section{Workflow and Web Applications}

In Figure 4 the high level integration of the Granta MI database, extended to include both real and virtual data, was described. Here, new workflow and associated web applications will be discussed in more detail. Figure 8 illustrates this workflow/communication, schematically, among three individual platforms; the end user machine, the MI server and the high performance compute (HPC) server. The separation of each function enables the development of a workflow that will encompass a corporate/global installation (a single point installation merely being a special case) of the ICME implementation wherein the end user machine, MI server, and HPC server can be located anywhere. The interface between the user and Granta MI (i.e., MI:Server) is accomplished via two new Web applications. The first, named MAC demonstrator, provides the interface for defining/executing a given composite analysis, whereas, the second, named MAC Composite Model, provides easy viewing of resulting information within the composite Table with little or no knowledge of Granta MI required. The overall workflow described in Figure 8 captures GRC's MAC/GMC simulation data, with full model pedigree, within the context of a Granta MI installation. 


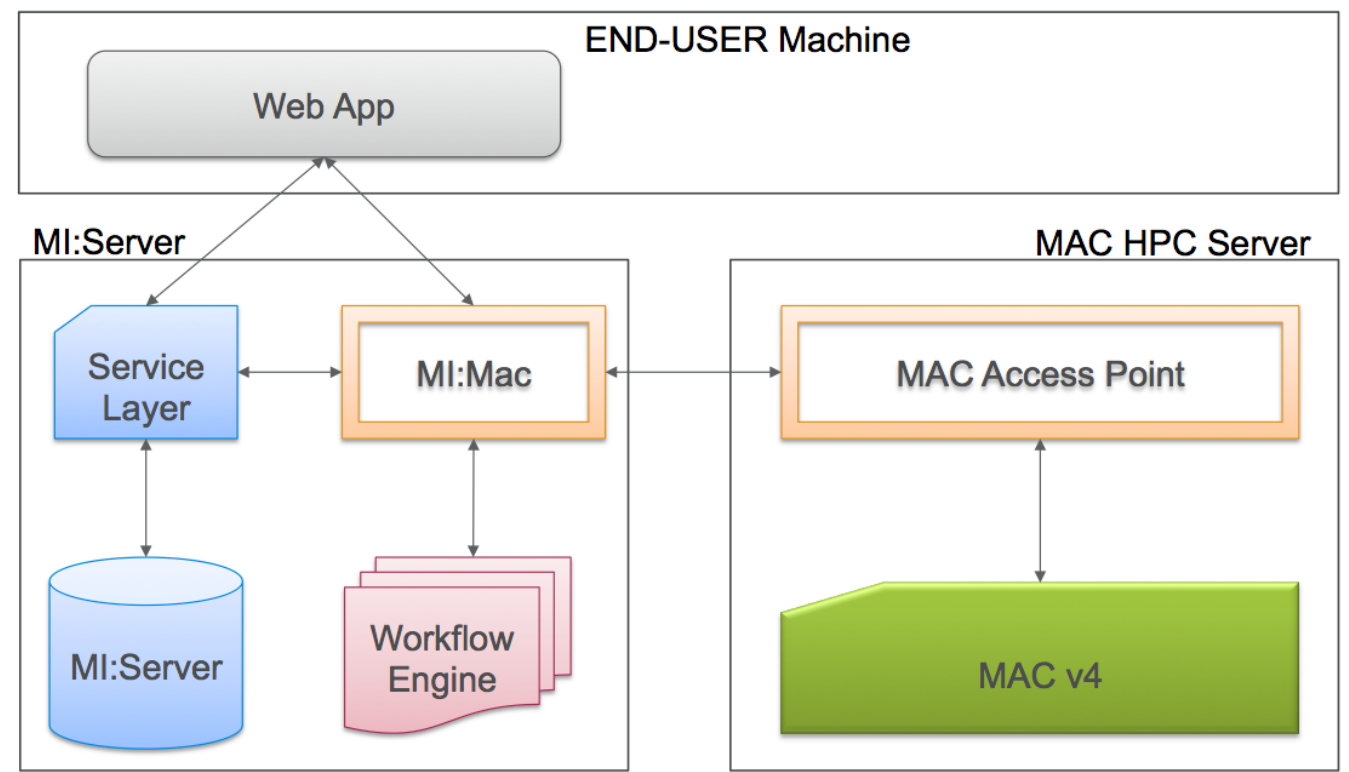

Figure 8 Schematic describing the information flow between three key workflow components.

The MAC demonstrator web application, illustrated in Figure 9, enables multiple composite analyses (e.g., the computation of effective properties) to be performed with no user intervention beyond the declaration of fiber and matrix constituents, analysis temperatures $\left(30^{\circ} \mathrm{C}\right.$ to $\left.120^{\circ} \mathrm{C}\right)$ of interest, and variation in fiber volume fraction $(10 \%-$ $80 \%$ ) at each temperature. Figure 9a illustrates the definition of a Graphite/Epoxy (polymer matrix composite, PMC) problem. Note in the pane on the right side of Figure 9b, a point and click ability to define constituent material parameters is enabled via access to the Deformation Table within GRC's Granta MI database. Although merely a prototype demonstration here, this web app will be fully configurable (in terms of type of problems (microstructure, loading, etc.) and type of analysis (linear or nonlinear) to be performed) in the future. To enable this general configurability, MI:Mac was developed to interact with web apps, Granta MI (through its already established Service Layer), and out of the box (OOTB) workflow engine. The MI:Mac module also writes the associated input file for the external analysis tool (in this case $\mathrm{MAC} / \mathrm{GMC}$ ) and captures the corresponding calculated output results as well as all metadata. Again, to enable full generality, a MAC access point module was constructed which drives the interaction between MI:MAC and the executable third party software package, in this case, MAC/GMC. This is the main point of entry to manage the execution of MAC/GMC. This code records the incoming requests, manages the disk storage, utilizes the HTTP service to execute the MAC/GMC jobs, and manages the retention of input/output files by assigning unique job identifiers to both input and output files generated. Then it passes the requested results back to the MI:Mac module. This separation of functionality in the workflow is important to enable future integration of the outlined workflow with other third party analysis software tools, in that specific input/output requirements can be isolated to substitutable modules of code, while not duplicating common functions between toolsets. 


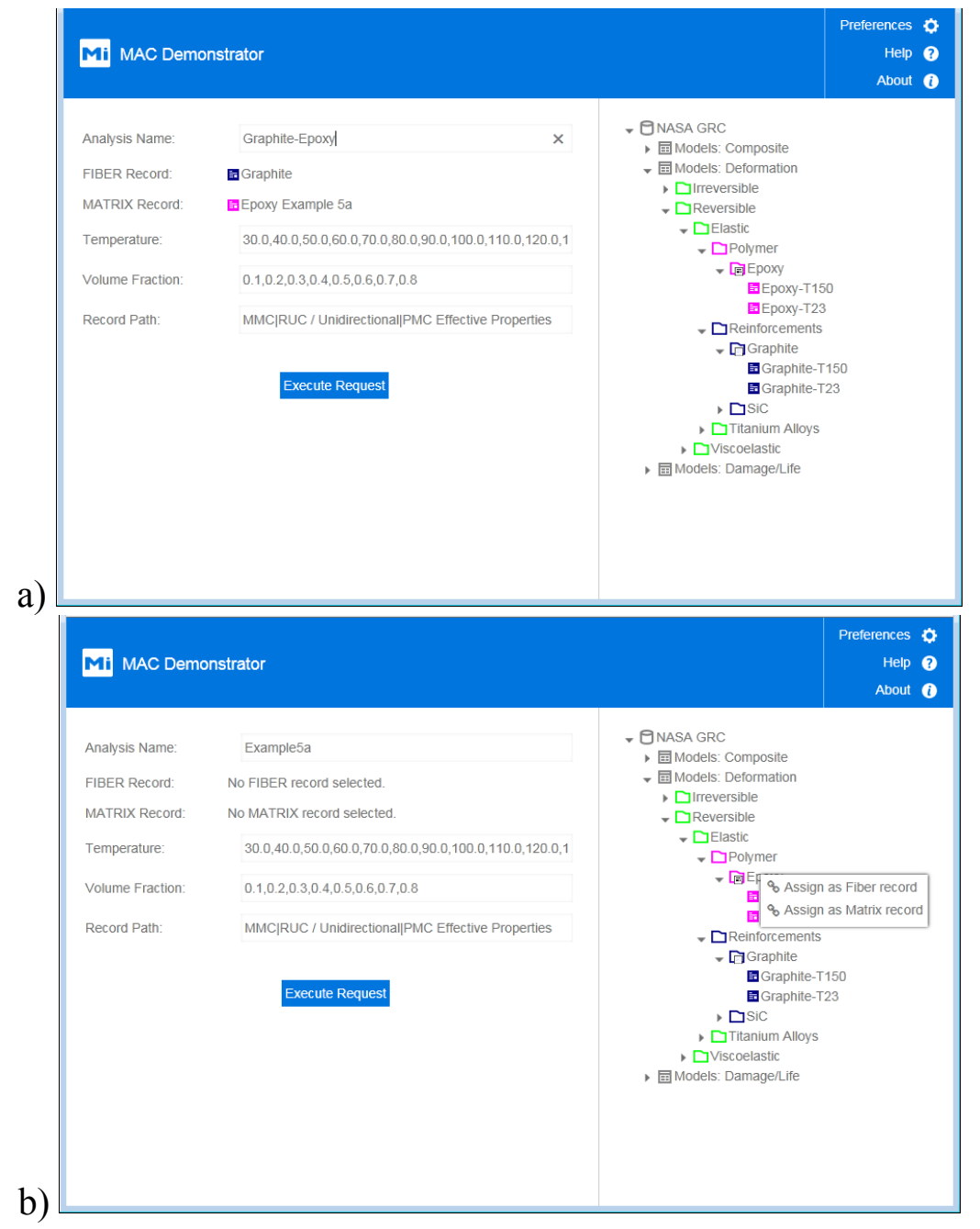

Figure 9 An example Graphite/Epoxy problem is defined using MAC Demonstrator.

Results from a metal matrix composite (MMC) example (defined in Fig. 10) are provided in Figures 11 and 12. Figure 11 shows one of nine resulting records that were automatically loaded into NASA GRC's database by the execution of the problem set defined in Figure 10. In Figure 11, additional attributes associated with output results, not identified earlier in the Composite Model Table (see Table 1) have been included, specifically the: 1) screen output results (stdout.std), 2) MAC/GMC output file which mimics the input file, 3) effective properties output file newly generated to aid in interfacing MAC/GMC with MI:Mac, and 4) all individual macro and/or micro output files requested by user. The insert, outlined in red, in Figure 11 illustrates the fact that these files now contain unique embedded identifiers to ensure pedigree integrity. Figure 12 further illustrates that for the present demonstration, all effective properties were written to their own discrete effective property attributes (instead of the effective Tabular attribute previously defined, see Table 1), while the effective stress-strain response was stored in the predefined response attributes, see insert outlined in red in Figure 12. Finally, Figure 13 shows all nine effective stress-strain curves resulting from the automated analyses performed using the MAC:Demonstration application, using the comparison chart feature within Granta MI. However, not all users will be familiar with the use of Granta MI, therefore a simple results viewing application (MAC:Composite Model, Figures 14 and 15) was customized for viewing information within the Composite Model Table. 


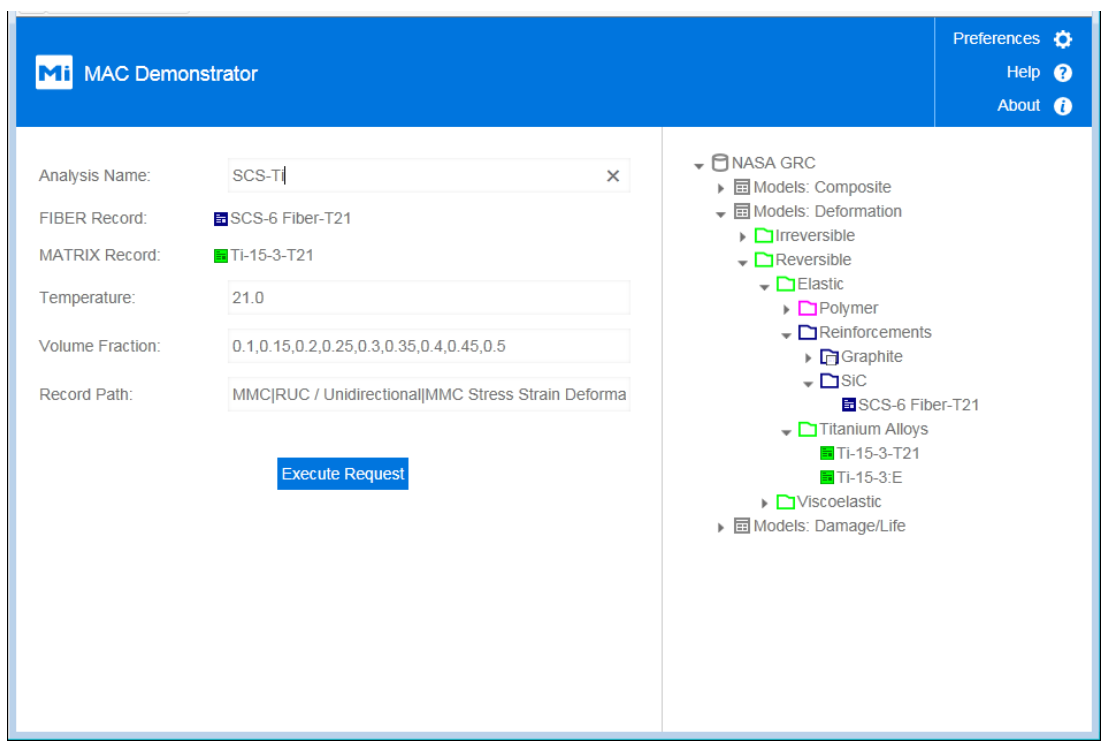

Figure 10 An example MMC (SCS-6/Ti-15-3) problem is defined using MAC Demonstrator

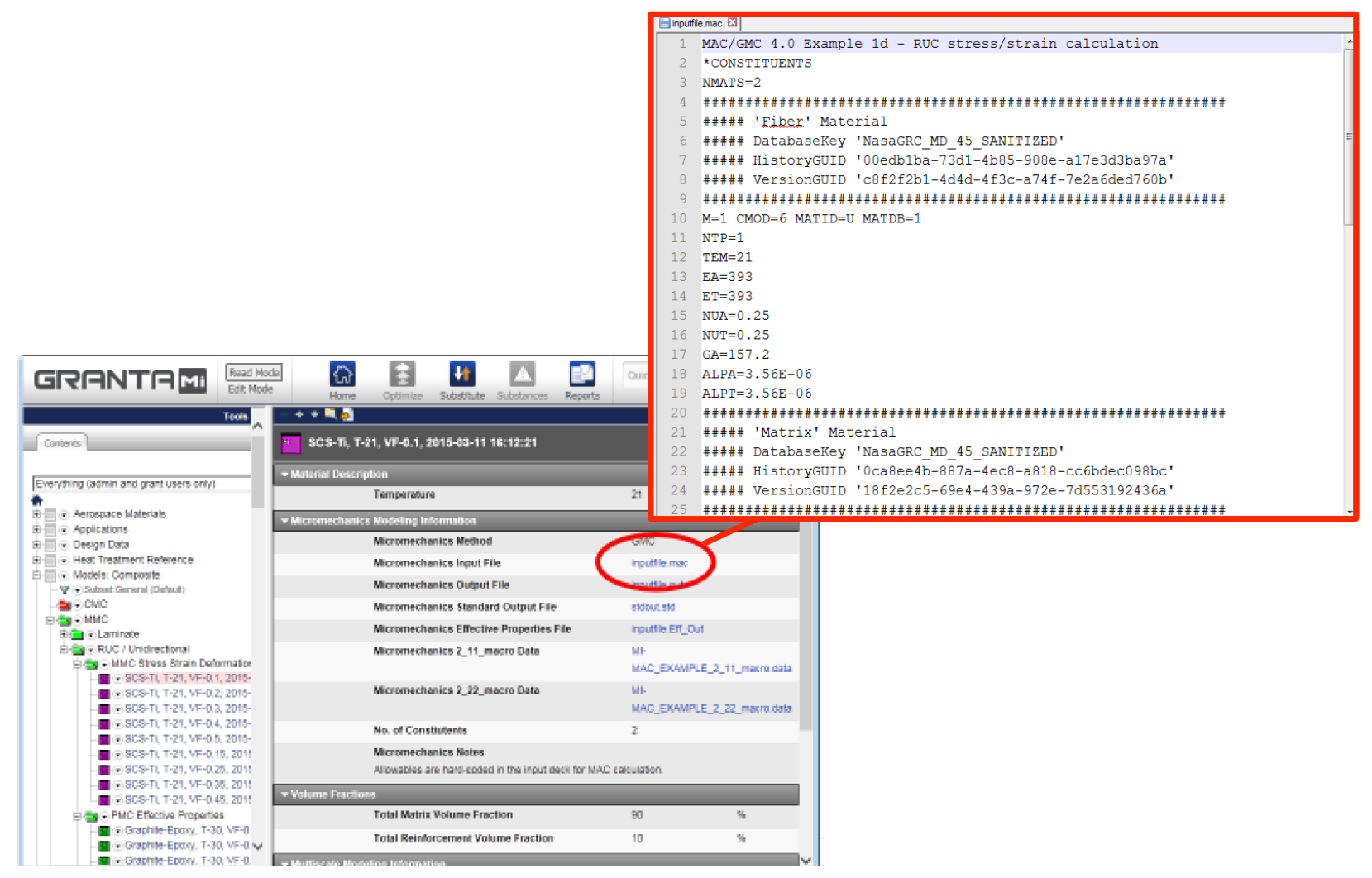

Figure 11 Illustration of detailed record within NASA GRC's database 


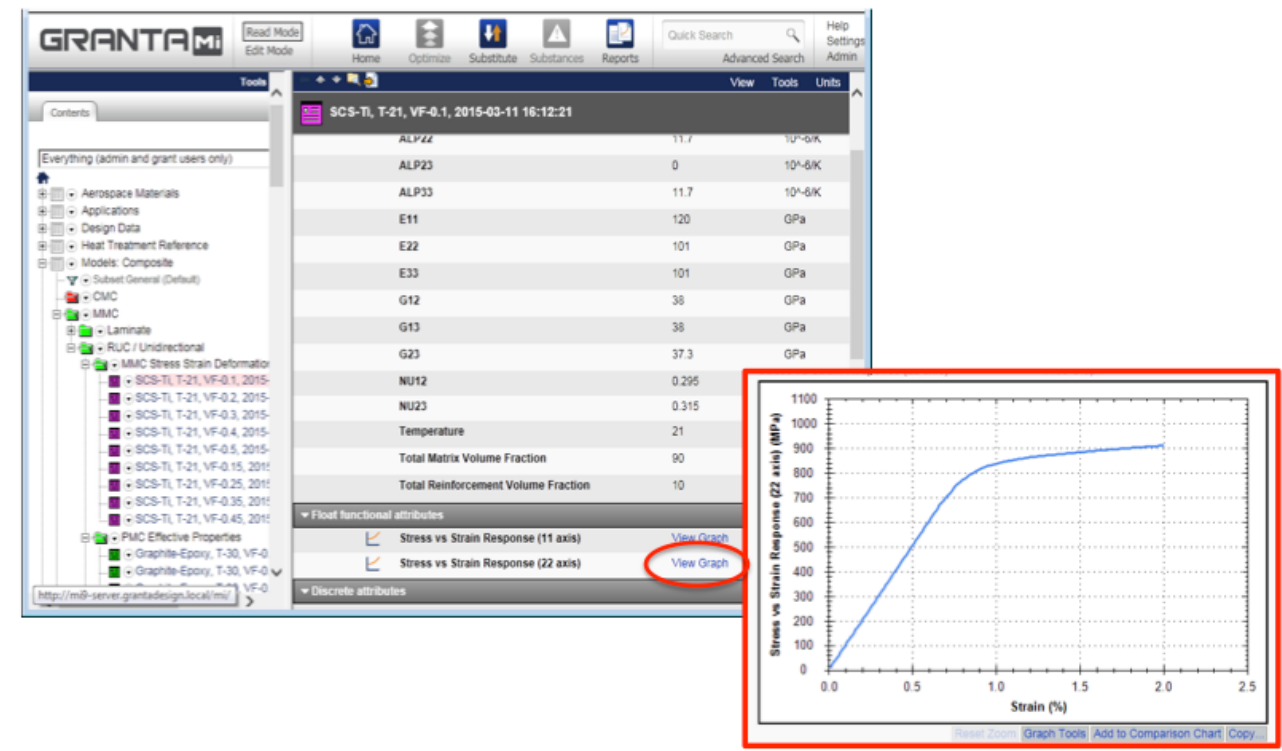

Figure 12 Illustration of detailed record within NASA GRC's database, continued from Fig. 11

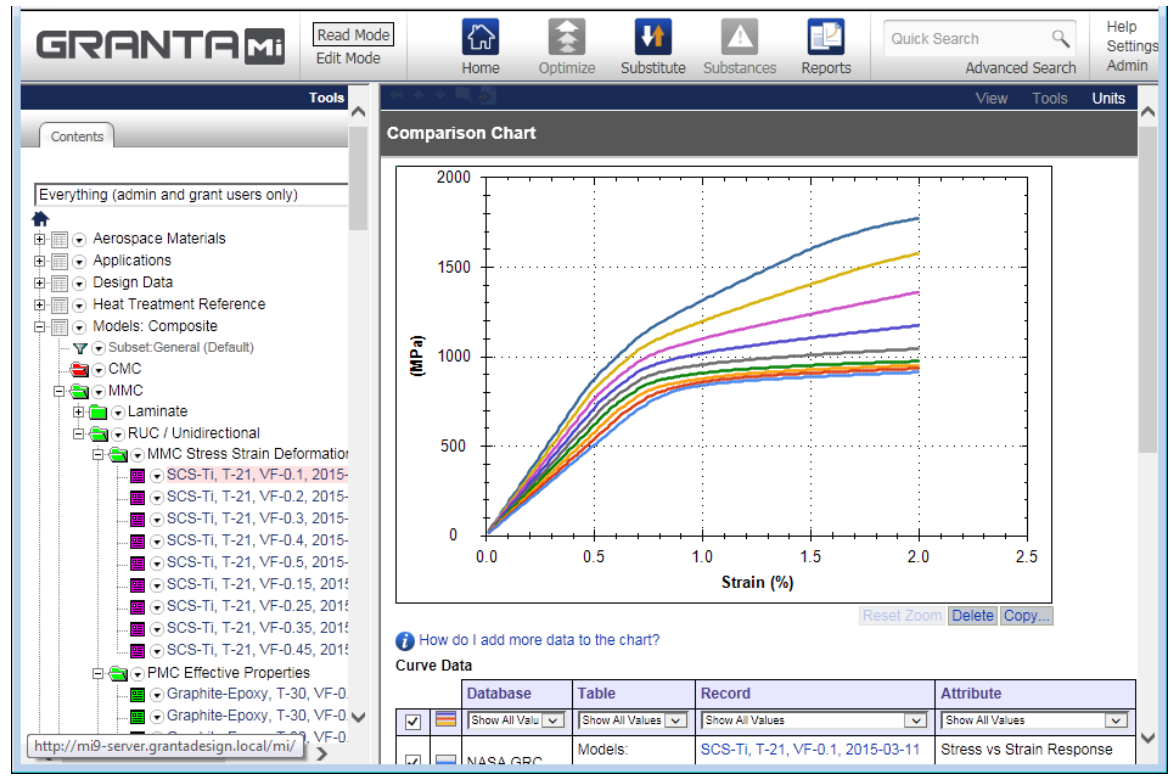

Figure 13 Illustrates how all nine stress-strain response curves, associated with SCS-6/Ti-15-3, at RT can be plotted via the comparison chart feature within Granta MI.

The MAC:Composite Model web application enables one to view results within the Composite Model Table residing in NASA GRC's Granta MI database with little or no prior knowledge of Granta MI. The Web app is configurable based on selected preferences. The left hand side of Figure 14 illustrates how ranges for a variety of different attributes are set using either discrete values or slider bars. These ranges limit the material records shown on the right side of the window (see Figure 14). The actual values and columns shown on the right hand side (List) are set by selecting from a menu of options shown in the insert of Figure 14. Figure 15 shows the resulting right hand side (List tab) when Name, No. of Constituents, Temperature, and Total Reinforcement Volume Fraction are 
selected. Finally, Figure 16 demonstrates that search results can also be displayed graphically using the chart mode. Note that if one hovers the mouse over a given point (circled in red, see Figure 16) the name of the record appears and if one then double clicks on the data point another window opens showing the different attributes stored in this record (see insert in Figure 16 outlined in red).

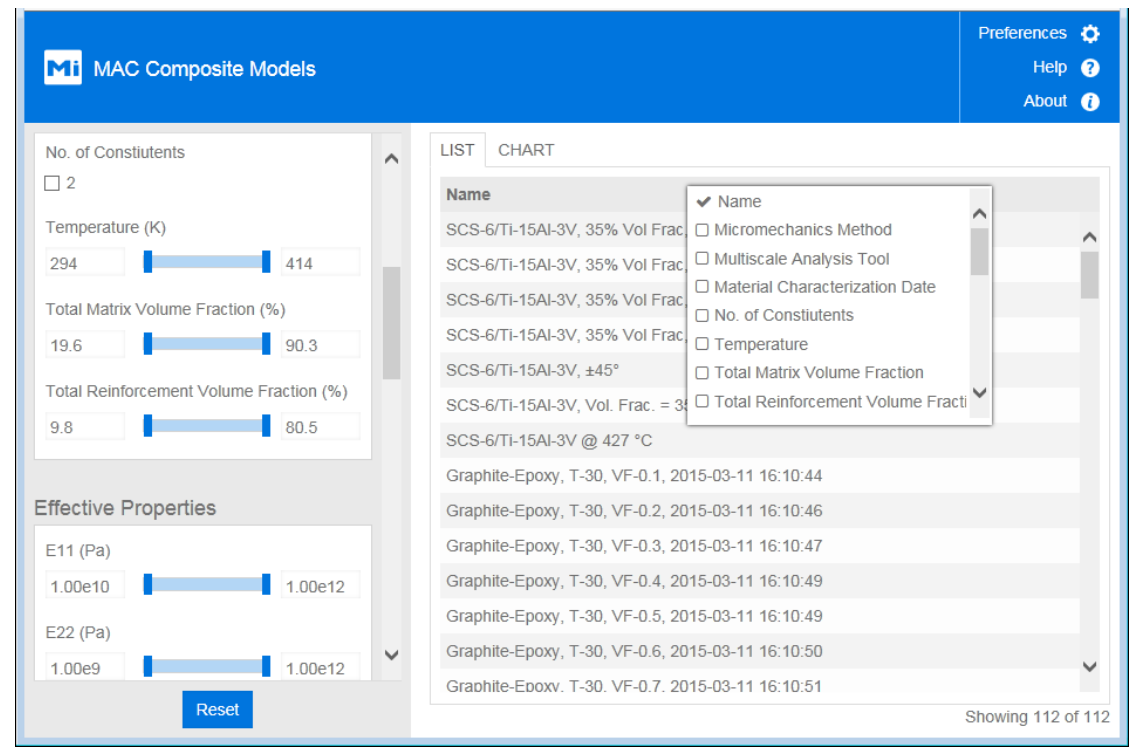

Figure 14 Illustration of MAC Composite Model web application: Configuration Insert

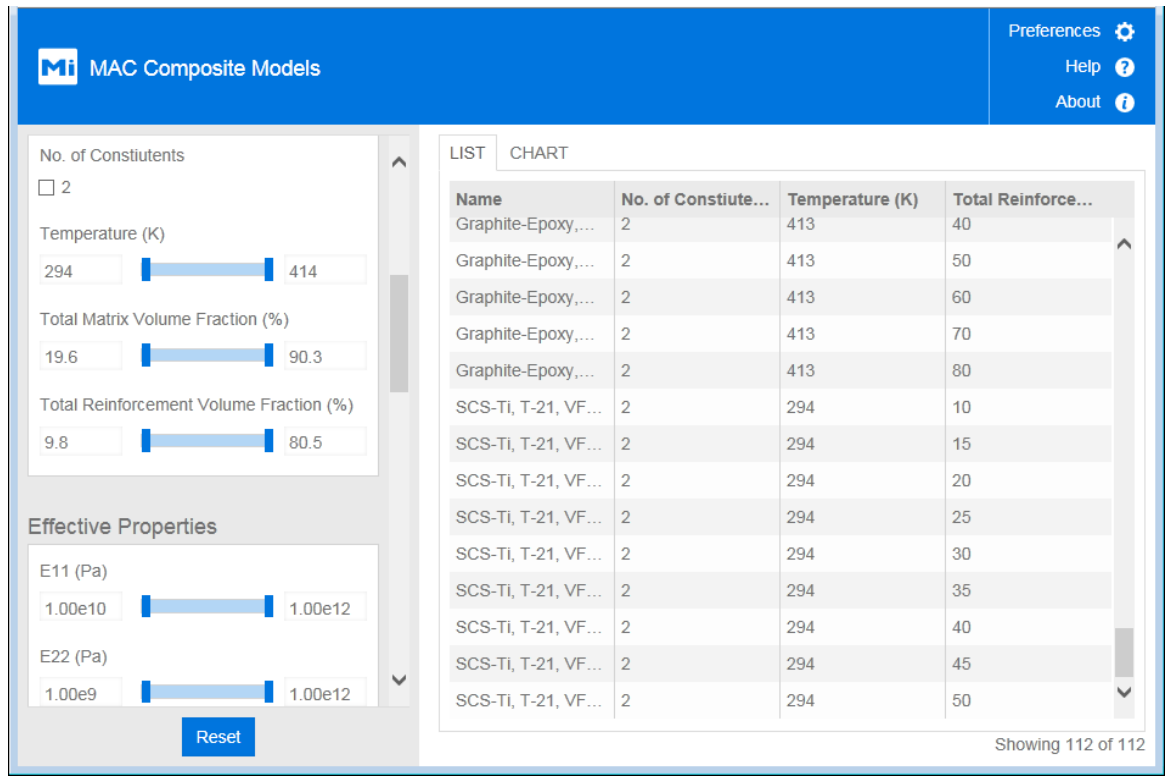

Figure 15 Illustration of MAC Composite Model web application: List window reconfigured 


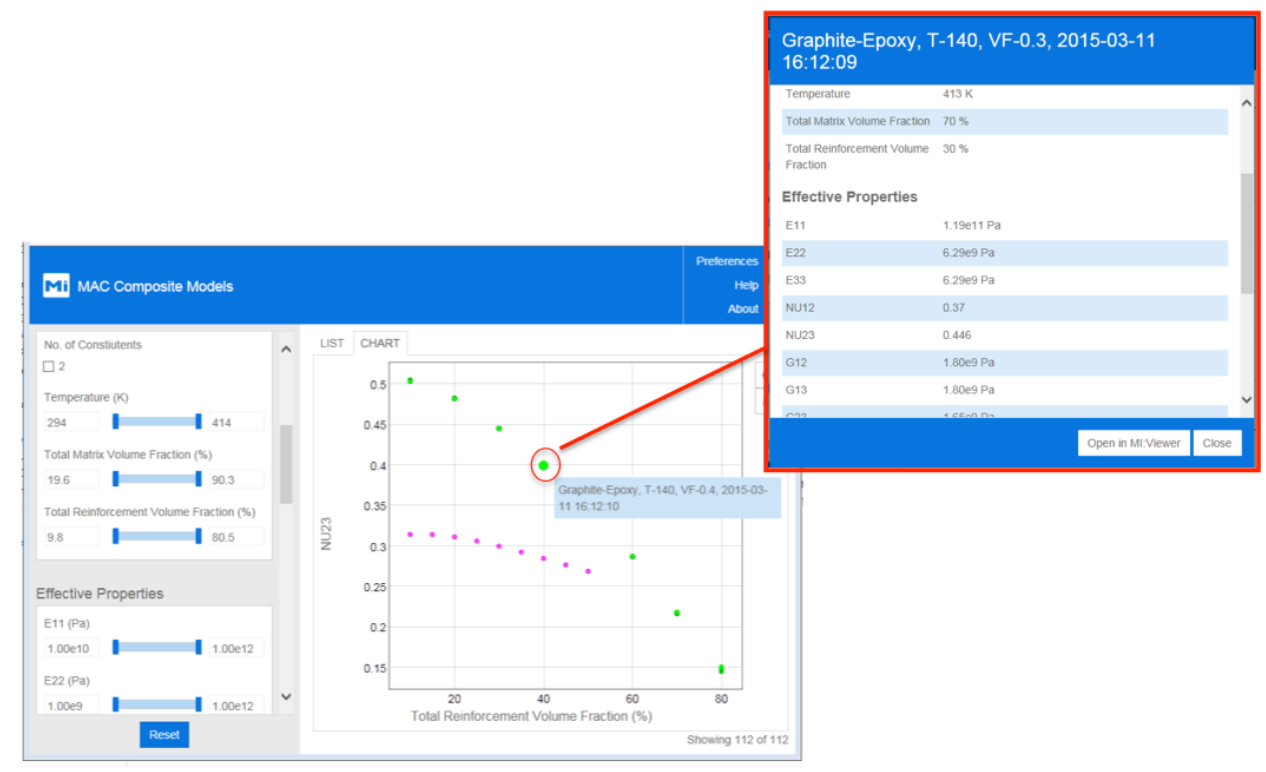

Figure 16 Illustration of MAC Composite Model web application: Chart Display

By automatically storing simulation result data, as illustrated herein for MAC/GMC, the data are not only available for future use, but their complete pedigrees are also captured, stored, and made searchable. Therefore, even years later, the input data, and even the version of the simulation code, for each predicted value, are readily available. This real time traceability renders the data perpetually useful across an organization without any need to repeat simulations. Clearly, as multiscale simulations become more complex and more expensive, this virtual data paradigm combined with a digital data storage culture, can provide tremendous returns on investment.

\section{Conclusions}

As models become more accurate, their complexity tends to increase, as they rely less and less on simplifying assumptions. This complexity drives the need for more data to be measured, predicted, compared, stored, and tracked. The goal of ICME, to link models at multiple scales, drives these same needs and underscores the value of a robust information management system. Often overlooked as a "mere database," this information management system should be viewed as a "necessary" or an "enabling" infrastructural aspect to ICME. In this paper, we have taken the next step in implementing a robust information management system by outlining the workflow and demonstrating the associated web applications necessary to enable linkage of third party model simulation software (with full model pedigree storage) to such an information management system (Granta MI) at two levels of scale. Such linkage opens up the design space and enables seamless and rapid connection of experimental data with virtual (simulation) data at various levels of scale, thereby enabling fit for purpose material tailoring.

\section{References}

1. National Research Council, Integrated Computational Materials Engineering: A Transformational Discipline for Improved Competitiveness and National Security, the National Academies Press (Washington, DC, 2008)

2. Will Marsden, David Cebon and Elizabeth Cope, "Managing Multi-Scale Material Data For Access Within ICME Environments," in Tools, Models, Databases, and Simulation Tools Developed and Needed to Realize the Vision of Integrated Computational Materials Engineering, S.M. Arnold and T. Wong (Eds.); ASM International, (Materials Park, OH, 2011).

3. S.M. Arnold, Holland, F.A., Gabb, T., Nathal, M. and Wong, T.; "The Coming ICME Data Tsunami and What Can Be Done”, 54th AIAA/ASME/ASCE/AHS/ACS Structures, Structural Dynamics, Materials Conference, Boston, MA (April 23-27, 2013).

4. D. Cebon and M.F. Ashby, "Engineering materials informatics,” MRS Bulletin, Vol. 31, No. 12, pp. 1004-1012, 2006.

5. Arnold, S.M., Holland, F. and Bednarcyk, B.A.; (2014). Robust Informatics Infrastructure Required For ICME: Combining Virtual and Experimental Data, 55th AIAA/ASMe/ASCE/AHS/SC Structures, Structural Dynamics, and Materials Conference, National Harbor, Maryland, 13 - 17 January 2014, AIAA-2014-0460. 
6. Arnold, S.M., Holland, F.A., Bednarcyk, B.A., and Pineda, E.J.; "Combining Material and Model Pedigree is Foundational to Making ICME a Reality”, IMMI, 4:4 DOI 10.1186/s40192-015-0031-2, 2015

7. Open MDAO, see http://openmdao.org

8. Granta Design Limited, http://www.grantadesign.com/, accessed on March 19, 2013.

9. Official website of Materials Data Management Consortium, http://mdmc.net, accessed on March 19, 2013

10. Collier Research Corporation makers of HyperSizer; www.HyperSizer.com

11. Simulia Abaqus, a Dassault Systemes subsidiary; http://www.3ds.com/products-services/simulia/overview/

12. Aboudi, J., Arnold, S.M., and Bednarcyk, B.A. (2013) Micromechanics of Composite Materials: A Generalized Multiscale Analysis Approach, Elsevier, Oxford, UK.

13. Sullivan, R. W. and Arnold, S. M., "An Annotative Review of Multiscale Modeling and its Application to Scales Inherent in the Field of ICME," Models, Databases, and Simulation Tools Needed for the Realization of Integrated Computational Material Engineering, S. M. Arnold and T. T. Wong, Eds., ASTM International, Materials Park, OH, pp. 6-23, 2011.

14. Bednarcyk, B. A., and Arnold, S. M., "MAC/GMC 4.0 User's Manual - Keywords Manual," NASA/TM-2002-212077/VOL2, 2002a.

${ }^{15 .}$ Bednarcyk, B. A., and Arnold, S. M., "MAC/GMC 4.0 User's Manual - Example Problems Manual," NASA/TM-2002212077/VOL3, 2002b.

${ }^{16 .}$ Bednarcyk, B. A., and Arnold, S. M., "A Framework for Performing Multiscale Stochastic Progressive Failure Analysis of Composite Structures," Proceedings of the 2006 Abaqus User's Conference, May 23-25, Boston, MA, 2006. 